\title{
Study of One of the Largest Space Weather Event of Solar Cycle 23: Events of November 2004
}

\author{
Nisha Patel $^{1 * 2}$, Ajaysinh K. Jadeja ${ }^{1}$ \\ ${ }^{1}$ Sir P. T. Sarvajanik College of Science, Surat-395 001, India \\ ${ }^{2}$ Shri J J T University, Rajsthan. - 333 001, India \\ ${ }^{1}$ Gujarat Ayurved University, Jamnagar-361 008, India
}

\begin{abstract}
In this paper, we discuss the solar origin and interplanetary consequences of the coronal mass ejections of November, 2004 which was one of the largest space weather events of Solar Cycle 23 and also one of the most difficult periods to forecast. Nine halo coronal mass ejections (CMEs), interacting on their way through the interplanetary medium and forming two complex geo-effective ICME structures which reached to the Earth on 7-8 and 9-10 November, whereas the later, more rapid CMEs seems to be non geoeffective. The Dst reached to $-373 \mathrm{nT}$ for the duration of 7--8 November and -289 nT for the duration of 9-10 November and the Kp index was 9 for 2-3 hour periods during every storms.
\end{abstract}

Keywords: CME- Coronal mass ejection, solar cycle, Geomagnetic effect, ICME, interplanetary magnetic field - IMF

\section{Introduction}

The fundamental role played by CMEs as the drivers of enhanced interplanetary and geomagnetic activity is now quite well accepted [Schwenn, 1986; Tsurutani et al., 1988; Gosling, 1993]. To establish a physical relationship between the solar origin and the final geomagnetic effect, hence it is required to monitor the CMEs right from the solar surface through the interplanetary medium till they arrive at the earth. More recently, with the launch of Solar and Heliospheric Observatory (SoHO), it has become possible to track a CME from the solar surface using Extreme Ultraviolet Telescope (EIT) images.

In order to address the fundamental problem of prediction of geomagnetic storms, one needs to examine the halo CMEs, first observed by Howard et al. [1982]. The halo CMEs may be directed either towards or away from the earth and appear as expanding, circular brightenings surrounding the occulter of the coronagraph. In this paper we examined CMEs during initial 10 days of November, 2004 and its consequent geomagnetic storms.

\section{Observation and Analysis}

Activity of November 2004 was originated in AR 0696 with two spurts: the first one was frail with a several halo CMEs and low level SEP flux. The chief activity started as the AR was at N09W17 on $7^{\text {th }}$ November with an X 2.0 flare, and a CME with speed $1759 \mathrm{~km} / \mathrm{s}$. There were some halo CMEs on $6^{\text {th }}, 7^{\text {th }}, 9^{\text {th }}$, and $10^{\text {th }}$ November, 2004 and all of them were associated with a particle event. The halos were extremely fast, with speeds of $818 \mathrm{~km} / \mathrm{s}$ ( $6^{\text {th }}$ November), $1759 \mathrm{~km} / \mathrm{s}\left(7^{\text {th }}\right.$ November), $2000 \mathrm{~km} / \mathrm{s}$ ( $9^{\text {th }}$ November) and $3387 \mathrm{~km} / \mathrm{s}\left(10^{\text {th }}\right.$ November). The very last CME had the maximum average sky-plane speed in solar cycle 23. Also, the solar wind speed remained prominent for few days in association with 4 shocks and 3 magnetic clouds. The magnetic clouds resulted in two super extreme storms (-373 nT and -289 nT). The former storm is undoubtedly associated with the 6th November halo. This halo was furthermore preceded by one more halo of moderate speed $(818 \mathrm{~km} / \mathrm{s})$ from the same region. The magnetic storm must have been formed by the complex structure of the two CMEs. Fig. 1 shows a LASCO image showing the compound structure consisting of the two CMEs and the GOES plot showing various eruptions. There was lone shock associated with the two CMEs, but the ICME was rather extended. The second storm on $10^{\text {th }}$ November is most probably due to the halo on 7th November at 16:54 UT. There were two shocks prior to the storm-causing magnetic cloud. 


\section{International Journal of Science and Research (IJSR) \\ ISSN (Online): 2319-7064}

Index Copernicus Value (2013): 6.14 | Impact Factor (2014): 5.611

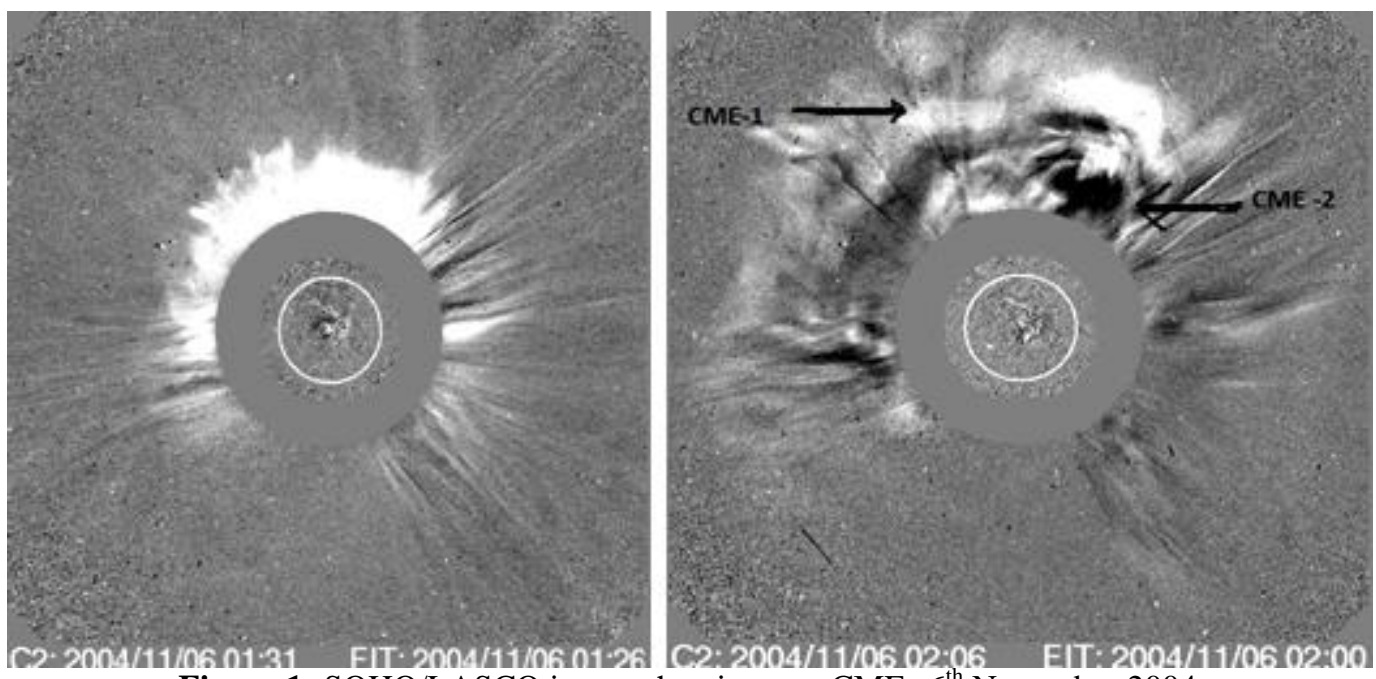

Figure 1: SOHO/LASCO image showing two CMEs $6^{\text {th }}$ November 2004
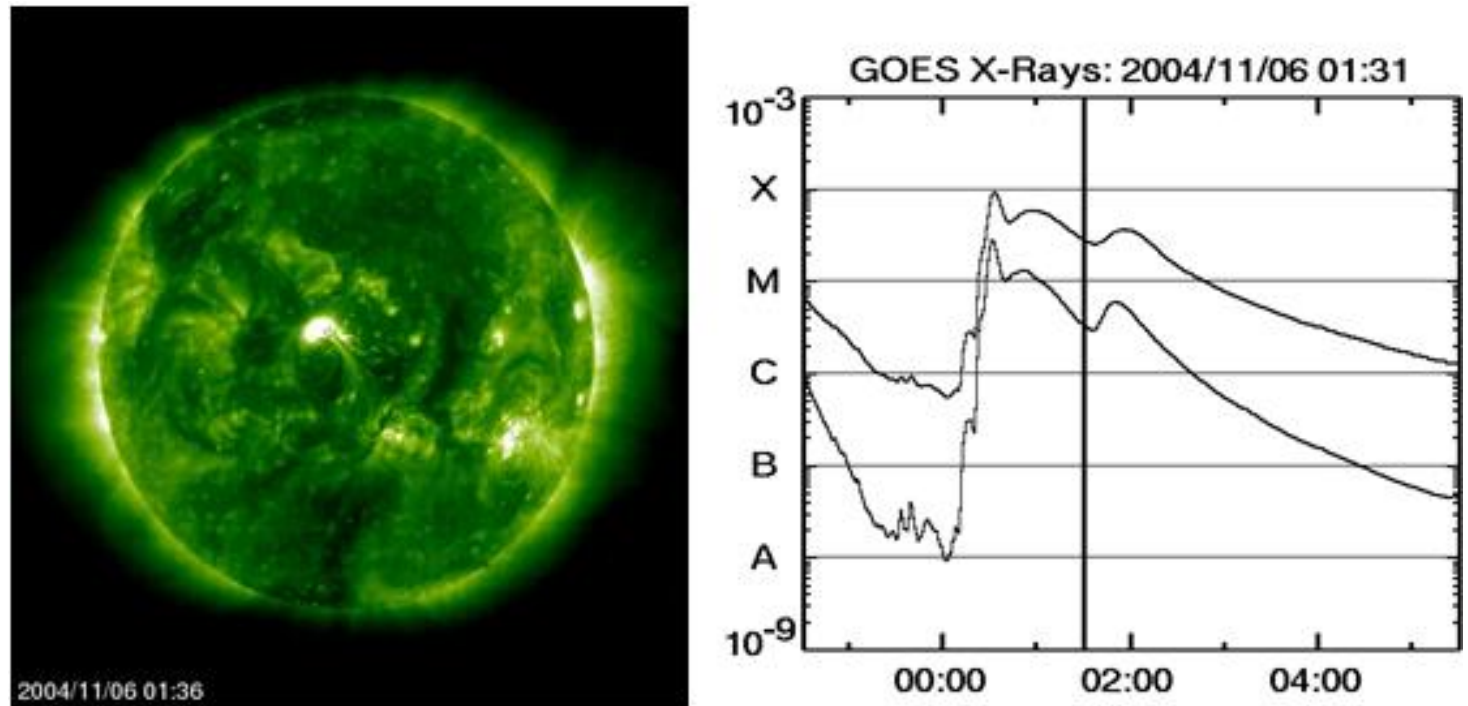

Figure 2: The flare was observed in EIT on $6^{\text {th }}$ November, 2004 and GOES X-ray light curve indicating multiple eruptions

Table 1: List of Halo CMEs Produced by NOAA AR 10696 in November 2004

\begin{tabular}{|c|c|c|c|c|c|c|}
\hline CME NO. & Date & Time (UT) & Type of CME & $\begin{array}{c}\text { Associated Flare } \\
\text { Location }\end{array}$ & $\begin{array}{c}\text { Associated } \\
\text { Flares }\end{array}$ & Speed of CMEs \\
\hline 1 & 03-Nov & $03: 54$ & Partial & N09E45 & M1.6 & 750 \\
\hline 2 & 03-Nov & $16: 06$ & Full & N11E40 & M5.0 & 1016 \\
\hline 3 & $04-N o v$ & $09: 04$ & Partial & N08E28 & C6.3 & 635 \\
\hline 4 & $04-N o v$ & $23: 30$ & Partial & N11E19 & M2.5 & 1053 \\
\hline 5 & $06-$ Nov & $01: 31$ & Partial & N10E08 & M9.3 & 960 \\
\hline 6 & $07-N o v$ & $17: 06$ & Full & N09E17 & X2.0 & 1770 \\
\hline 7 & $08-N o v$ & $04: 06$ & Full & N08E28 & C7.9 & 520 \\
\hline 8 & 09-Nov & $17: 26$ & Full & N07E51 & M8.9 & 1853 \\
\hline 9 & 10-Nov & $02: 26$ & Full & N09E49 & X2.5 & 1975 \\
\hline
\end{tabular}

In Table 1 lists of all CME with associated flares and locations of them on the solar disk for the period of 3-10 November 2004. Though the solar sources of the space weather events of November were relatively clear, their consequent interplanetary structures became complex once the CME left the Sun.
There were two immense jumps in geomagnetic activity in November 2004 which presumably caused by multiple interacting CMEs making it complicated to forecast or even to establish the direct connection between an individual $\mathrm{CME}$ and its geophysical effect, if any.

\section{Interplanetary Consequences:}


International Journal of Science and Research (IJSR)
ISSN (Online): 2319-7064

Index Copernicus Value (2013): 6.14 | Impact Factor (2014): 5.611

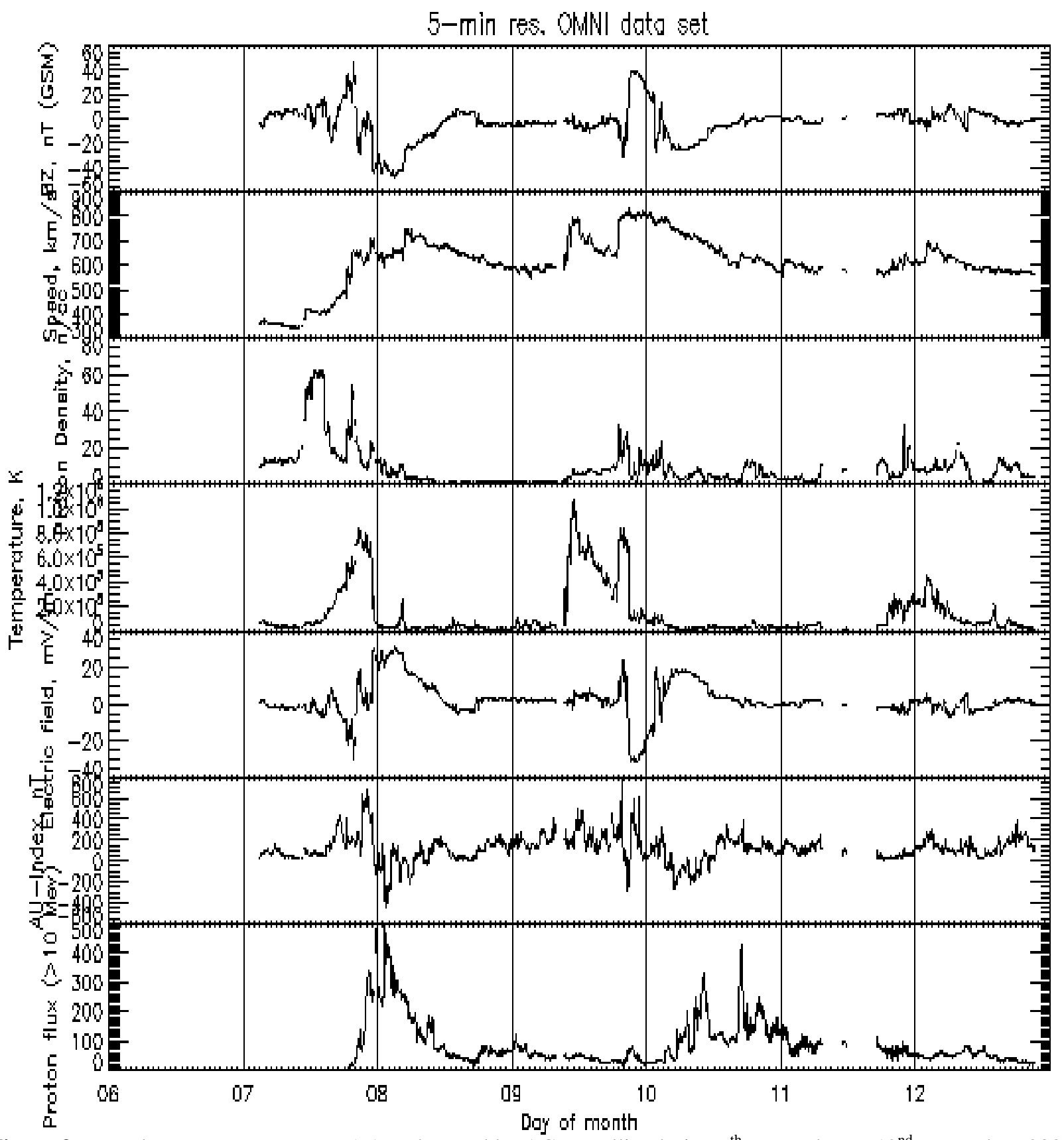

Figure 3: Interplanetary parameters at 1 AU observed by ACE satellite during $6^{\text {th }}$ November to $12^{\text {nd }}$ November, 2004

The tentative scenario for the propagation of nine halo CMEs and identification of the interplanetary structures can be described as follows. Most probably, the first CME missed the Earth, since no distinctive signature was found in the ACE solar wind data, probably as its origin was located far away from the central meridian. The subsequent five halo CMEs may correspond to the 5 shocks those have been observed on $7^{\text {th }}$ and $9^{\text {th }}$ November. The weak and slow full halo CME (Table-1) was perhaps swept up by the following rapid ones and together resulted in the sixth shock.

The CMEs presumably interacted en route from the Sun to the Earth, and the effect of their interactions was seen at ACE as a complex interplanetary CME (ICME) on 7-8 November, comprising one MC (magnetic cloud) and three shocks, and a $2^{\text {nd }}$ ICME on 9--10 November, comprising one MC and two shocks. Both periods contained elongated intervals of strong (i.e., >-50 nT) southward interplanetary magnetic field (IMF). Involving these two diverse structures, the solar wind persist to have a high speed whereas other solar wind parameters and the IMF became relatively quiet. Also, a $3^{\text {rd }}$ disturbed period in the interplanetary medium can be identified during the period of 11-- 12 November with lesser amplitude and no considerable southward turning.

The energetic ( $>10 \mathrm{MeV}$ ) protons started to appear at GOES on 7 November with a sudden boost in the daily proton flux owing to the X2.0 flare (Figure 4.16 bottom panel). The increase on 10 November was possibly produced by the X2.5 flare. As a number of flares and CMEs were detected through a relatively short time interval, it was not all the time possible to distinguish the different sources of the energetic particles. Proton fluxes stayed prominent until 17 November. 


\section{International Journal of Science and Research (IJSR) \\ ISSN (Online): 2319-7064}

Index Copernicus Value (2013): 6.14 | Impact Factor (2014): 5.611

Geomagnetic effect related to CMEs:

Interaction of the passing ICMEs and magnetosphere of the Earth produced intricate changes in geomagnetic field of the Earth. The Dst reached to -373 nT for the duration of 7--8 November (the $1^{\text {st }} \mathrm{CME}$ event) and $-289 \mathrm{nT}$ for the duration of 9-10 November ( $2^{\text {nd }} C M E$ event), and the Kp index was 9 for 2-3 hour periods during every storms. Daily Ap indices were 140 on $8^{\text {th }}$ November and 161 on $10^{\text {th }}$ November. Even though these indices are not remarkably large, the morphology of the geomagnetic field variations was complex.

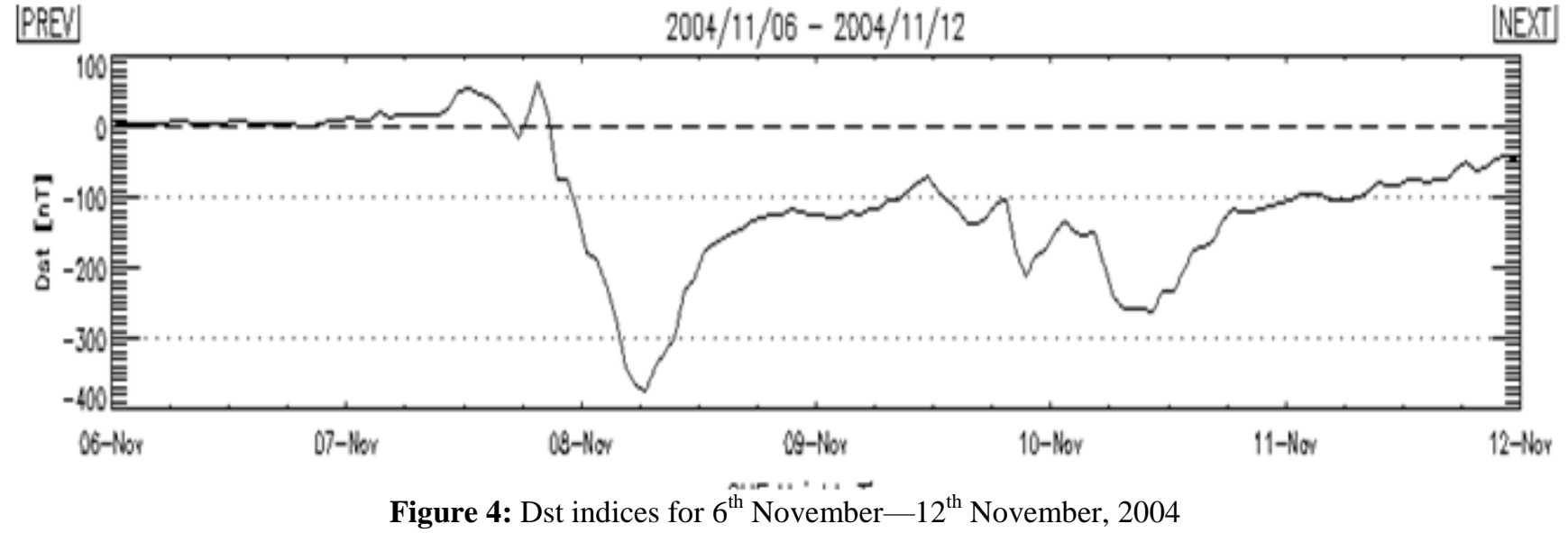

Here, the eruptive events of November 6 and 7 had been considered, which were associated with large scale dimming plus coronal waves and formed strong geomagnetic storms with $D$ st $=-373$ and $-289 \mathrm{nT}$, and the events of November 9 and 10, which implicated even larger-scale (global), homologous disturbances on the disk, since events representative of the activity outburst of November 2004.

\section{Conclusions}

Study of the space weather conditions for events of November 2004, based on real-time data, showed the earlier intricacy of the solar sources and constant interactions of the multiple (no less than nine) CMEs for the period of their propagation through the solar wind. These interactions led to the formation of only two complex geo-effective ICME structures which reached to the Earth on 7-8 and 9-10 November, whereas the later, more rapid CMEs seems to be non geo-effective. Ground observations showed that the first storm extended more quickly to lower latitudes, creating multifaceted events in the ionosphere, at the same time the second storm evolved further gradually and it was more regular in nature.

\section{References}

[1] Ajaysinh K. Jadeja, Effects of 18 November, 2003 Space Weather Events on the Interplanetary \& Ionospheric Medium and Geomagnetic Storms, Scientific Journal of Physical Science, Volume 4, Issue 1 2014, PP. 13-21.

[2] Kilcik et. Al., The Astrophysical Journal, 727:44 (6pp), 2011 January 20, doi:10.1088/0004-637X/727/1/44.

[3] J. Farrugia, Interplanetary coronal mass ejection and ambient interplanetary magnetic field correlations during the Sun-Earth connection events of OctoberNovember 2003, J. Geophys. Res, Vol. 110, A09S13, doi:10.1029/2004JA010968, 2005.

[4] Gosling, J. T. (1990), Coronal mass ejections and flux ropes in interplanetary space, in Physics of Flux Ropes,
Geophys. Monogr. Ser., vol. 58, edited by C. T. Russell, E. R. Priest, and L. C. Lee, pp. 343-364, AGU, Washington, D. C.

[5] N. Gopalswamy, Coronal mass ejections and other extreme characteristics of the 2003 October-November solar eruptions, Journal of Geophysical research,Vol. 110, A09S15, doi:10.1029/2004JA010958, 2005.

[6] N. Gopalswamy, P. K. Manoharan, and S. Yashiro (2003d), Comment on "Coronal mass ejections, interplanetary ejecta and geomagnetic storms" by H. V. Cane, I. G. Richardson, and O. C. St. Cyr, Geophys. Res. Lett., 30(24), 2232, doi:10.1029/2003GL017562.

[7] N. Gopalswamy, Halo coronal mass ejections and geomagnetic storms, Earth Planets Space, 61, 1-3, 2009.

[8] Nandita Srivastava and P. Venkatakrishnan, Relationship between CME speed and geomagnetic storm intensity, Geophysical research letters, VOL. 29, NO. 9, 1287, 10.1029/2001GL013597, 2002 1On lien from Indian Institute of Astrophysics, Bangalore.

[9] P. K Manoharan, , N. Gopalswamy, S. Yashiro, A. Lara, G. Michalek, and R. A. Howard (2004), Influence of coronal mass ejection interaction on propagation of interplanetary shocks, J. Geophys. Res., 109, A06109, doi:10.1029/2003JA010300.

[10] Tsurutani, B. T., W. D. Gonzalez, G. S. Lakhina, and S. Alex (2003), The extreme magnetic storm of $1-2$ September, 1959, J. Geophys. Res., 108(A7), 1268, doi:10.1029/2002JA009504.

[11]Webb, D., and J. Allen: Space Weather, 2, S03008, 2004.

[12] Zhang, J., M. W. Liemohn, J. U. Kozyra, B. J. Lynch, and T. H. Zurbuchen (2004), A statistical study of the geoeffectiveness of magnetic clouds during high solar activity years, J. Geophys. Res., 109, A09101, doi:10.1029/2004JA010410. 